\title{
KARP HARDNESS OF A GUARDING SET IN DIGRAPH
}

\author{
THINH D. NGUYEN
}

\section{Definiton And Claim}

Our problem is to decide whether a digraph has a guarding set of size at most $k$. Definitions are following.

A digraph $G(V, A)$ has $V(G)$ as its vertex set and $A(G)$ as its arc set. A guarding set $S \subseteq V(G)$ is a set of vertices such that:

- For each vertex $v \in V(G)$, some of its incoming neighbor $u$ (i.e. $(u, v) \in A(G)$ ) is included in $S$ (i.e. $u \in S$ ) and some of its outgoing neighbor $w$ (i.e. $(v, w) \in A(G))$ is included in $S$ (i.e. $w \in S$ ).

- In case of empty incoming neighborhood or empty outgoing neighborhood, the vertex $v$ itself must be included in $S$. In other word, we may think that the incoming neighborhood and outgoing neighborhood of $v$ both include $v$.

Informally, imagine a facility in which one can enter at some entry points and exit at some exit points, a guarding set for this facility needs to be placed at least at some entry point (maybe where one can enter secret area of the facility) and at some exit point (maybe where one can bring out the secrets from the secret areas inside). Hope that this suffices to explain the terminology.

Our problem is now formally defined:

Definition 1. Digraph Guarding Problem:

Input: An oriented graph $G=(V, A)$ and a natural number $k$

Output: YES if $G$ has a guarding set of size $k$, otherwise No

It is NP-complete (it is obviously in NP) by reduction from the dominating set problem whose hardness was proven in [1].

Claim 2. We have that Dominating Set $\leq_{p}$ Digraph Guarding Problem

\section{Reduction from Dominating Set}

Given an instance $G=(V, E)$ of the dominating set problem, split each vertex $v$ into two vertices $v_{\text {in }}$ and $v_{\text {out }}$, and for each edge $(u, v) \in E$, construct two edges $\left(u_{\text {out }}, v_{\text {in }}\right)$ and $\left(v_{\text {out }}, u_{\text {in }}\right)$. We claim that $G$ has a dominating set of size $k$ iff the newly-constructed graph (say $G^{\prime}$ ) has a guarding set of size $2 k$.

If $G$ has a dominating set $S$ of size $k$, easy to see $\left\{v_{\text {in }} \mid v \in S\right\} \cup\left\{v_{\text {out }} \mid v \in S\right\}$ is a guarding set of $G^{\prime}$, which has size $2 k$.

Key words and phrases. digraph, guarding, in-neighbor, out-neighbor.

Perebor. 
On the other hand, suppose $G^{\prime}$ has a guarding set $S^{\prime}$ of size $2 k$. Let $S_{\text {in }}^{\prime}=$ $S^{\prime} \cap\left\{v_{\text {in }} \mid v \in V\right\}$ and $S_{\text {out }}^{\prime}=S^{\prime} \cap\left\{v_{\text {out }} \mid v \in V\right\}$, then either $S_{\text {in }}^{\prime}$ or $S_{\text {out }}^{\prime}$ must has size at most $k$. Without loss of generality, say $\left|S_{\text {in }}^{\prime}\right| \leq k$, then $S=\left\{v \mid v_{\text {in }} \in S_{\text {in }}^{\prime}\right\}$ is a dominating set of $G$. This means $G$ has a dominating set of size $k$.

\section{Conclusion}

Garey and Johnson [1] shape their theory based on previous primal works of Cook, Levin and Karp. Johnson [2] moves on with the guide to this theory. As long as we study a mathematical conjecture, we should encourage ourselves to have enough labour hours on popular mathematics books like these. Then, reading some articles on theory of computing like [3] is a good practice. Only after that, could we think of the ultimate final for all mathematical sciences.

\section{REFERENCES}

1. Michael R. Garey, David S. Johnson, Computers and Intractability: A Guide to the Theory of NP-Completeness

2. David S. Johnson, The NP-Completeness Column: An Ongoing Guide

3. Phan Dinh Dieu, Le Cong Thanh, Le Tuan Hoa, Average Polynomial Time Complexity of Some NP-Complete Problems, Theor. Comput. Sci. 46(3): pp.219-237 (1986)

Email address: kosmofarmer@yandex.com 